\title{
Irish mothers' intentions to have daughters receive the HPV vaccine
}

\author{
Amanda Fahy B.A., Deirdre M. Desmond Ph.D. \\ Department of Psychology, John Hume Building, National University of Ireland \\ Maynooth, Maynooth, Co. Kildare, Ireland. \\ *Correspondence \& Requests for Reprints: \\ Dr Deirdre Desmond, Department of Psychology, John Hume Building, National \\ University of Ireland Maynooth, Maynooth, Co. Kildare, \\ E-mail: Deirdre.Desmond@ nuim.ie
}

Telephone: 353-1-708 6479 Fax: 353-1-708 4767 


\begin{abstract}
Most cases of cervical cancer are associated with human paillomavirus (HPV) types 16 and 18. This study examined the effect of message framing on mother's intentions to obtain HPV vaccination for their teenage daughters and investigated predictors of HPV vaccination intentions. Seventy two mothers of daughters in the 8 to 16 years age group were randomly assigned to read either a gain-framed (describing the benefits of receiving the vaccine) or a loss-framed (describing the costs of not receiving the vaccine) message and completed measures assessing their intention to have their daughter/s vaccinated, normative beliefs, attitudes and perceived behavioural control. Awareness of HPV was low but intentions to vaccinate were high. There was no effect of message frame on vaccination intentions. Attitudes toward HPV vaccination and the influence of both peers and medical professionals are important factors in HPV vaccine acceptability.
\end{abstract}




\section{Introduction}

Human papillomavirus (HPV) infections are the most commonly diagnosed sexually transmitted viral infections. Most of the burden of high risk HPV infection is associated with cervical cancer [1]. Cervical cancer is the second most common cancer affecting women aged 15-44 years in the European Union; approximately $73 \%$ of cervical cancer cases in Europe are caused by HPV types-16 and -18 [2]. In 2006, two forms of HPV vaccine were licensed in Europe, the U.S. and elsewhere. The WHO and the European Centre for Disease Control, amongst others, support routine HPV vaccination for young women prior to sexual debut.

As preadolescent children are the primary target for HPV vaccination parents' willingness to have their children vaccinated is critical to uptake. Concerns have been raised about the vaccine's acceptability to parents and regarding appropriate strategies to motivate parents to immunise their children [3]. Communicating relevant health information is often the first step in promoting particular health behaviours. Health information can stress the potential benefits of performing the behaviour (gain-framed) or can emphasis the possible costs of not performing the healthy behaviour (loss-framed). The influence of message frame is dependent on the type of behaviour being promoted [4]. Prospect Theory (PT) [5] suggests that when making decisions people are risk averse when considering gains while risk seeking when considering losses. In the context of health, detection behaviours (e.g. screening) are generally perceived as more risky than prevention behaviours (e.g. sunscreen use). People are more open to taking risks when faced with the possibility of loss, hence loss-framed appeals should be beneficial for motivating detection behaviours. Conversely, gain-framed appeals should facilitate 
prevention behaviours which are associated with minimal risk because their goal is to avoid disease [4]. Accordingly, it has been hypothesized that promoting vaccination, a preventive behaviour, should be facilitated by gain framed appeals. However, research has yielded inconsistent results [6-11]. The first aim of the current study is to examine the effectiveness of gain- versus loss-framed messages in promoting parental HPV vaccination uptake intentions.

The second aim is to identify predictors of HPV vaccination intentions. According to the Theory of Planned Behaviour (TPB) [12] performance of a particular behaviour is predicted by the intention to perform the behaviour which in turn is a function of attitude, normative beliefs and perceived behavioural control [12]. Thus individuals are likely to intend to vaccinate their daughters if they believe the behaviour will lead to valued outcomes, that significant others think they should carry out the behaviour and that they have the necessary resources or opportunities to perform the behaviour. Here we examine the efficacy of the TPB in the context of mother's intentions to have their daughters receive the HPV vaccine.

\section{Methods}

\section{Participants and Procedure}

A convenience (non-random) sample of mothers with at least one daughter between the ages of 8 and 16 years was recruited via snowball sampling (i.e. initial contacts were asked to refer potential participants to the study). Participants were resident within the school catchment area of Maynooth town, a university town located in north County Kildare, Ireland. Participation was voluntary and was not incentivised; all those approached agreed to participate. 
After providing written informed consent participants were given a selfcompletion study booklet. Initially, they answered questions regarding age, family composition, cervical cancer and vaccination. Next they read a one-page summary describing HPV infection and either a gain- or loss-framed message about HPV vaccination. For example, the gain frame message stated: 'by choosing to have your child vaccinated with the HPV vaccine she may be less likely to develop cervical cancer'. The corresponding statement in the loss framed message stated: 'by choosing not to have your child vaccinated with the HPV vaccine she may be more likely to develop cervical cancer'. After reading the information, participants completed assessments of TPB variables.

\section{Measures}

Pre-intervention measures. Age, family history of cervical cancer (yes/no), and personal usage of cervical cancer screening services (at least every 3 years; yes/no) were assessed. Participants also rated their attitudes to vaccines in general on a 5-point scale from positive (1) to negative (5).

Post-intervention measures. As a manipulation check participants indicated whether the frame focussed on the benefits of receiving the HPV vaccination (=7) or the risks of not receiving the HPV vaccination (=1). They also indicated the extent of their agreement with the statements: (a) I learned a lot from reading the information; and (b) I found the information about the HPV vaccine to be informative. Ratings were on a 7-point scale ( $1=$ disagree strongly to $7=$ agree strongly $)$. 
HPV vaccination intentions were assessed using three items rated on 7point scales. Participants indicated their agreement with the statements: 'I will consent to having my daughter(s) vaccinated with the HPV vaccine if offered by a health care provider within the next two years'; and 'I expect to get my daughter vaccinated with the HPV vaccine' (disagree strongly/agree strongly). The third item required participants to indicate the likelihood that they would "consider getting the HPV vaccine' for their daughter (very likely/very unlikely). Average scores on these three items were used to create a scale of HPV vaccine acceptance (Cronbach's alpha = .748). Higher scores indicate stronger intention to vaccinate.

Attitudes towards having one's daughter vaccinated against HPV were assessed using three bipolar semantic differential scales (responsible/irresponsible; harmful/beneficial; worthless/useful). Items were averaged to obtain a scale score ( $\alpha$ $=.797)$.

Normative beliefs were assessed using two items: (a) 'most people important to me would want me to get my daughter(s) vaccinated'; and (b) 'My decision to have my daughter(s) vaccinated would be influenced by recommendations made by healthcare professionals' (disagree strongly $=1$ /agree strongly $=7$ ). The internal reliability estimate for these items was unacceptably low $(\alpha=.466)$, therefore these questions were considered individually in the regression model.

A single item assessed perceived behavioural control (PBC) 'I am confident that I could have my daughter(s) vaccinated with the HPV vaccine if I want to' $($ disagree strongly $=1$ /agree strongly $=7)$. 


\section{Data analyses}

Differences in pre-intervention measures between the gain and loss framed message groups were assessed using independent $t$ tests (continuous variables) and chi squared analyses (categorical variables). Average vaccination uptake intention scores were compared using an independent $t$ test. The effects of frame on perceived HPV vaccine risk, the single item measures of normative beliefs and $\mathrm{PBC}$ were tested using multivariate analysis of variance (MANOVA). Bivariate associations between vaccination intentions and predictor variables were assessed using Pearson productmoment correlations. Multivariate linear regression analysis was used to determine significant independent predictors of vaccination intentions.

\section{Results}

Seventy-two mothers of female children between the ages of 8 and 16 years participated in the study. Mean (M) age was 41.56 years (Standard deviation (SD) 5.81; range $29-53$ ). Sixty-five per cent of the sample had one daughter in the 8 to 16 year age group (collectively participants had 97 daughters, mean age 13.3 years); $87.5 \%$ reported no family history of cervical cancer; $81.9 \%$ reported regular attendance for cervical cancer screening and $33.3 \%$ indicated they had not heard of HPV. Attitudes towards vaccination in general were favourable $(\mathrm{M}=1.73, \mathrm{SD}=$ $1.13)$.

Of the 72 participants, 36 read a gain-framed message and 36 read a lossframed message. There were no significant differences between framing groups in terms of attitudes towards vaccines in general, family history of cervical cancer, age or personal usage of cervical cancer screening (all p's > .3). A manipulation check 
indicated that participants in the loss-frame condition reported that the HPV information focussed more on the costs of not getting the HPV vaccine ( $\mathrm{M}=3.97$, SD $=2.02)$ than on the benefits of getting the vaccine $\left(t_{(70)}=3.45, \mathrm{p}=.001\right.$; gain-frame: $\mathrm{M}=5.58, \mathrm{SD}=1.93)$. Across framing conditions participants reported similar evaluations of the extent to which the information provided was informative and educational (all p's > .6).

Framing effects

Mother's intentions to have their daughters vaccinated were high and did not vary by framing condition $(\mathrm{p}=.397$; gain: $\mathrm{M}=5.9, \mathrm{SD}=1.3$; loss: $\mathrm{M}=5.62, \mathrm{SD}$ = 1.4). MANOVA revealed no significant main effect of frame on attitudes, perceived behavioural control and normative beliefs $(\mathrm{F}[1,70]=.19, \mathrm{p}=.69)$.

[Insert table 1 about here]

Prediction of HPV vaccination intentions

Descriptive data and correlations between variables are shown in Table 1. Regression of intentions onto attitude, normative beliefs, and PBC accounted for $69.5 \%$ of the variability in intentions $\left(\mathrm{F}_{(4,71)}=38.23, \mathrm{p}<.001\right)$, see Table 2. Stronger intentions to have daughters receive the vaccination were related to positive attitudes toward HPV vaccination, the influence of peer groups (normative belief item 1) and recommendations of healthcare professionals (normative belief item 2).

[Insert table 2 about here]

\section{Discussion}


The current study is the first to investigate HPV vaccine acceptability among Irish mothers. Awareness of HPV was low; nonetheless, mothers reported strong interest in HPV vaccination for their daughters and favourable attitudes towards vaccination in general. HPV vaccine uptake intentions were uniquely predicted by attitudes and normative beliefs. Both peer groups and medical professionals may play an important role in promoting HPV vaccine acceptance. Attitude was the dominant predictor of behavioural intention. Both positive and negative messages affect attitudes regarding vaccination. If HPV vaccination is to be promoted parents will require education regarding the benefits and safety of the vaccine.

While this study provides an interesting first step in understanding Irish parents' willingness to accept the HPV vaccination, limitations of the study should be noted and findings interpreted with caution. First, the small, non-random sample means that the generalizeability of the findings to the wider population is unknown. Research using larger representative samples is necessary to determine whether these findings generalise to the wider Irish population. Future studies should also assess HPV vaccine acceptability among fathers. Second, the current study examined the influence of a subset of possible influential variables on intentions and did not assess actual vaccination behaviour. 


\section{References}

1. European Centre for Disease Prevention and Control, 2009. Guidance for the introduction of HPV vaccines in EU countries. Stockholm.

2. European Cervical Cancer Association, 2009. HPV vaccination across Europe. Brussels.

3. Ogilvie G, Remple V, Marra F, et al., 2007.Parental intention to have daughters receive the human papillomavirus vaccine. CMAJ, 177(12): 15061512.

4. Rothman AJ, Salovey P, 1997.Shaping perceptions to motivate healthy behavior: The role of message framing. Psychol Bull, 121: 3-19.

5. Kahneman D, Tversky A, 1979.Prospect theory: an analysis of decision making under risk. Econometrica, 47(2): 263-291.

6. Abhyankar P, O'Connor DB, Lawton R, 2008. The role of message framing in promoting MMR vaccination: Evidence of a loss-frame advantage. Psychol Health Med, 13(1): 1 - 16.

7. Gerend MA, Shepherd JE, 2007.Using message framing to promote acceptance of the human papillomavirus vaccine. Health Psychol, 26(6): 745752.

8. Gerend M, Shepherd J, Monday K, 2008.Behavioral Frequency Moderates the Effects of Message Framing on HPV Vaccine Acceptability. Ann Behav Med, 35(2): 221-229.

9. Ferguson E, Gallagher L, 2007.Message framing with respect to decisions about vaccination: The roles of frame valence, frame method and perceived risk. Br J Psychol, 98: 667-680. 
10. McCaul KD, Johnson RJ, Rothman AJ, 2002.The effects of framing and action instructions on whether older adults obtain flu shots. Health Psychol, 21(6): $624-628$.

11. Rothman AJ, Martino SC, Bedell BT, Detweiler JB, Salovey P, 1999. The systematic influence of gain- and loss-framed messages on interest in and use of different types of health behavior. Pers Soc Psychol Bull, 25(11): 13551369.

12. Ajzen I, 1991.The theory of planned behaviour. Organ Behav Hum Decis Process, 50(2): 179-211. 
Table 1: Means, standard deviations and correlations between study variables.

\begin{tabular}{lrcccc}
\hline & $\begin{array}{c}\text { Mean } \\
\text { (SD) }\end{array}$ & Intention & Attitudes & PBC & $\begin{array}{c}\text { Normative } \\
\text { belief 1 }\end{array}$ \\
\hline Intention & 5.76 & & & & \\
& $(1.38)$ & & & & \\
Attitudes & 6.16 & $.801^{* *}$ & & & \\
& $(1.06)$ & & & & \\
Perceived behavioural & 6.49 & $.442^{* *}$ & $.542^{* *}$ & & \\
control (PBC) & $. .79)$ & & & & \\
Normative belief 1 & 6.14 & $.617^{* *}$ & $.587^{* *}$ & $.483^{* *}$ & \\
& $(1.15)$ & & & & \\
Normative belief 2 & 6.10 & $.553^{* *}$ & $.543^{* *}$ & $.248^{*}$ & $.310^{* *}$ \\
& $(1.41)$ & & & & \\
\hline
\end{tabular}

** Probability value $(\mathrm{p})<0.01 ; * \mathrm{p}<.005$.

Normative belief 1: 'Most people important to me would want me to get my daughter(s) vaccinated'. Normative belief 2: 'My decision to have my daughter(s) vaccinated would be influenced by recommendations made by healthcare professionals'. 
Table 2: Multiple regression of intention on attitude, subjective norms and perceived behavioural control, perceived HPV vaccine risk and concerns about HPV vaccine impact on sexual behaviour.

\begin{tabular}{lcccr}
\hline Predictor & $\mathbf{R}^{2}$ & Standardised Beta & $\mathbf{t}$ & $\mathbf{p}$ \\
\hline Attitude & .695 & .590 & 5.85 & $<.001$ \\
Normative belief 1 & & .234 & 2.72 & .008 \\
Normative belief 2 & .168 & 2.09 & .040 \\
Perceived behavioural control & & -.032 & -.368 & .701
\end{tabular}

$\mathrm{p}=$ probability value

Normative belief 1: 'Most people important to me would want me to get my daughter(s) vaccinated'. Normative belief 2: 'My decision to have my daughter(s) vaccinated would be influenced by recommendations made by healthcare professionals'. 